\title{
Surface event rejection of the EDELWEISS cryogenic germanium detectors based on NbSi thin film sensors
}

\author{
S.Marnieros, L.Berge, A.Broniatowski, M.Chapellier, S.Collin, \\ O.Crauste, X.Defay, Y.Dolgorouky, L.Dumoulin, A.Juillard, \\ F.Lalu, C.Nones, E.Olivieri and the EDELWEISS Collaboration
}

Centre de Spectrométrie Nucléaire et de Spectrométrie de Masse, CNRS-IN2P3, Bât. 108, F-91405 Orsay Cedex, France

corresponding author: Stefanos.Marnieros@csnsm.in2p3.fr

\begin{abstract}
Near-surface-events are a major limitation to the performance of cryogenic massive germanium heat and ionisation detectors for dark matter search, due to their incomplete charge collection. We present here a powerful method of surface event identification based on the transient heat signal of a Ge bolometer, equipped with two NbSi high impedance thin film sensors. Calibration runs using electrons and low energy gamma particles from a ${ }^{109} \mathrm{Cd}$ source show highly effective surface event rejection down to the heat threshold energy. Neutron and gamma source calibrations were realised to get information on the fiducial volume of the Ge absorber. First results from low background data taking are discussed.
\end{abstract}

Received 22 July 2007 ; Accepted 15 September 2007

\section{INTRODUCTION}

Direct detection of WIMPs (Weakly Interacting Massive Particles) by cryogenic heat-ionization Ge detectors is often limited by near-surface events, characterized by incomplete charge collection ${ }^{1}$. A part of these events simulate nuclear recoils with a typical rate of $0.05 / \mathrm{kg} / \mathrm{d}$ above 30 $\mathrm{keV}$, and sets a lower limit of approximately $10^{-6} \mathrm{pb}$ for the WIMP-nucleon cross-section (EDELWEISS-I experiment ${ }^{2}$ ). Active rejection of near-surface events has been undertaken by many groups ${ }^{3,4}$ and seems very promising to go far beyond this limit.

\section{DETECTOR DESIGN}

The NbSi 212 detector presented here is fabricated in the CSNSM laboratory using high-vacuum electron-beam evaporators. The absorber consists of a $200 \mathrm{~g}, 10^{10} / \mathrm{cm}^{3}$ impurities, cylinder-shaped Ge crystal. Two $60 \mathrm{~nm}$ thick amorphous films of $\mathrm{Nb}_{\mathrm{x}} \mathrm{Si}_{1-\mathrm{x}}$ were deposited on the flat surfaces 


\section{S.Marnieros et al.}

of the absorber. A $50 \mathrm{~nm}$ thick a-Ge layer and $\mathrm{Nb}$ electrodes were evaporated prior to the NbSi sensors, followed by an a-Si layer on the top of the sensors to prevent oxidation. The NbSi films cover the whole central area of the Ge flat surfaces and act also as conductors for the charge signal. Enveloping guard electrodes on the side of the crystal detect events at the periphery, while two $500 \mu \mathrm{m}$ step interdigitized electrodes under each $\mathrm{NbSi}$ film probe its resistivity and measure simultaneously the centre charge signal (figure 1).

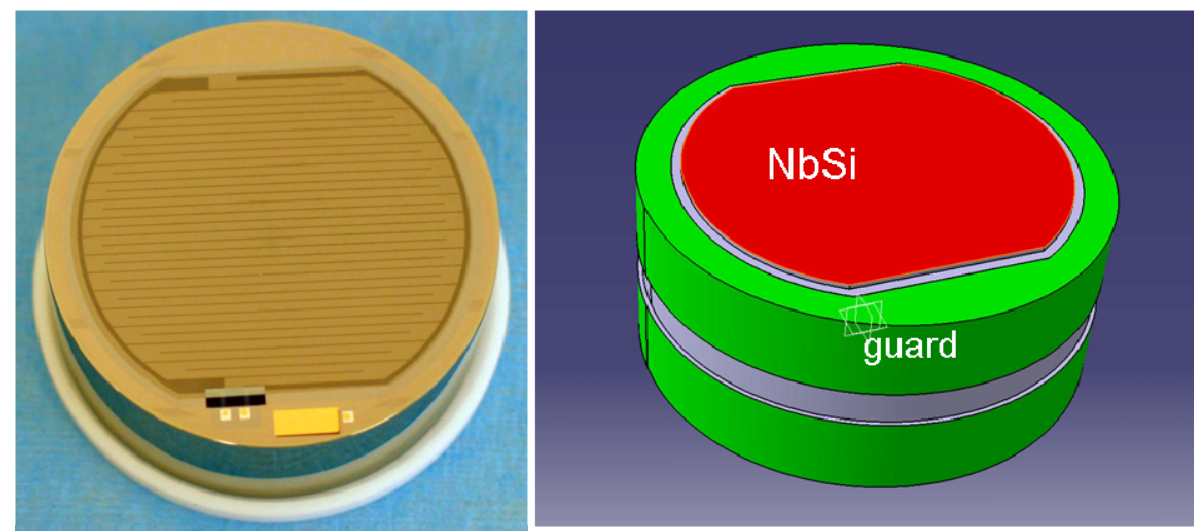

Fig.1. (Color on line) Photo of a NbSi 212 type bolometer with interdigitized comb geometry of the NbSi electrodes. The two faces of the detector are identical.

\section{NbSi SENSOR SIGNAL}

Typical NbSi pulses obtained for bolometer 212 are shown in figure 2 . The signal is composed of a transient part with high amplitude and relatively fast decay time and a thermal part characterized by a slower decay time. The transient signal results from absorption of high energy out-of equilibrium phonons by the $\mathrm{NbSi}$ film ${ }^{5}$. A fraction $\varepsilon$ of the deposited energy $E$ heats directly the sensor whose temperature rise $\Delta T_{\text {transient }}$ can be much higher than the thermal equilibrium one $\Delta T_{\text {thermal }}{ }^{6}$ :

$$
\Delta T_{\text {transient }}=\frac{\varepsilon E}{C_{e}} \quad \Delta T_{\text {thermal }}=\frac{E}{C}
$$

$C_{e}$ and $C$ are the electron heat capacity of $\mathrm{NbSi}^{7}$ and the total heat capacity of the detector.

The fraction $\varepsilon$ of high energy phonons absorbed by each NbSi film depends strongly on the position of the deposited energy into the detector. Events occurring close to the lateral surface of the Ge absorber (guard 
events) give approximately $\varepsilon=0.02$ while events just underneath the $\mathrm{NbSi}$ have $\varepsilon$ values typically 15 times higher (figure 2).
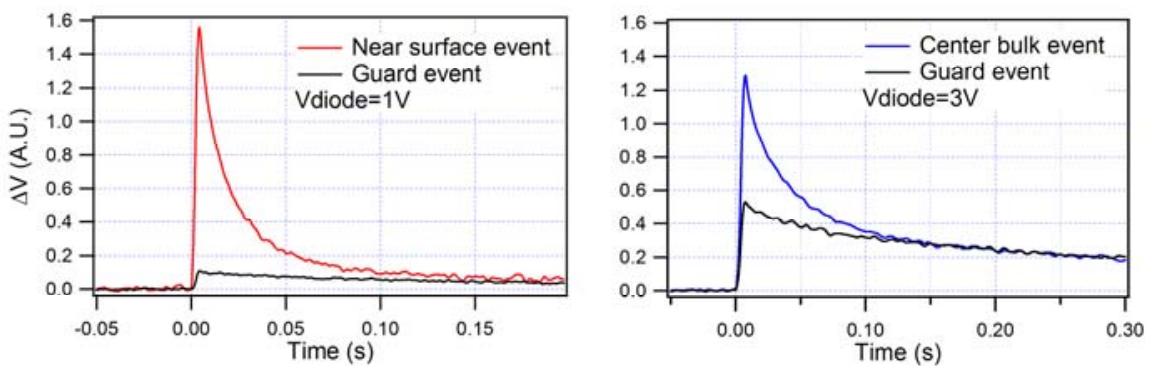

Fig. 2. (left) (Color on line) NbSi response to $22 \mathrm{keV} \mathrm{X}$-rays absorbed underneath the $\mathrm{NbSi}$, compared to guard events of the same energy (average over 100 events). (right) Difference between bulk events giving either centre or guard ionisation signal at $3 \mathrm{~V}$.

For a more accurate modelling of the athermal signal we must also take into account the high energy phonons produced by the charge drift $\left(\right.$ Luke effect ${ }^{8}$ ) as well as the recombination energy of the charges arriving at the NbSi film or the guard electrodes:

$$
\Delta \mathrm{T}_{\text {transient }}=\frac{\varepsilon_{\text {ph }} E_{\text {phonons }}+\varepsilon_{L} E_{\text {Luke }}+\varepsilon_{R} E_{\text {Recomb }}}{C_{e}}
$$

$\varepsilon_{\text {ph }} E_{\text {phonons }}$ is the part of the energy absorbed directly by the NbSi coming from the phonons initialy produced by the detected particle. In the present bolometer we estimate $0.02<\varepsilon_{p h}<0.3$ depending on the deposit distance from a given $\mathrm{NbSi}$ sensor.

$\varepsilon_{L}$ is similar to the $\varepsilon_{\mathrm{ph}}$ factor but concerns high energy phonons created by the Neganov-Luke effect ${ }^{8,9}$. Its value depends on the deposited energy position and particularly on the distance from the crystal z axis (very off-axis events have lower $\varepsilon_{L}$ ). For centre events, a part of these high energy phonons are created very close to the $\mathrm{NbSi}$ and will therefore be very effectively absorbed by the sensor. Our data are well fitted taking $\varepsilon_{L} \approx 0.04$ for centre events and $\varepsilon_{L}=0.02$ for guard events. The $\varepsilon_{R}$ factor indicates the percentage of the recoil energy effectively deposited in the NbSi sensor. Surface defects and the presence of an amorphous Ge or Si layer between the $\mathrm{Ge}$ crystal and the sensor can significantly reduce $\varepsilon_{R}$. Athermal signal modeling of $\gamma$ and neutron particles (figure 3 , right), suggests $\varepsilon_{R} \approx 0.2$ for centre events. 


\section{S.Marnieros et al.}

The resulting dependence of the heat transient signal to centre or guard events is clearly shown in figure 2 . For centre events at $3 \mathrm{~V}$ collecting bias, the FWHM resolution, using transient amplitude of both NbSi sensors, is close to $20 \%$ of the deposited energy (figure 3). Nevertheless the resulting signal histogram deviates from a Gaussian shape and some $13 \%$ of the events have significantly lower transient amplitudes, most probably due to the reduction of $\varepsilon_{p h}$ and $\varepsilon_{L}$ for far off z-axis centre events. The baseline resolution of the transient signal is $4 \mathrm{keV}$, compared to $15 \mathrm{keV}$ for the thermal part.
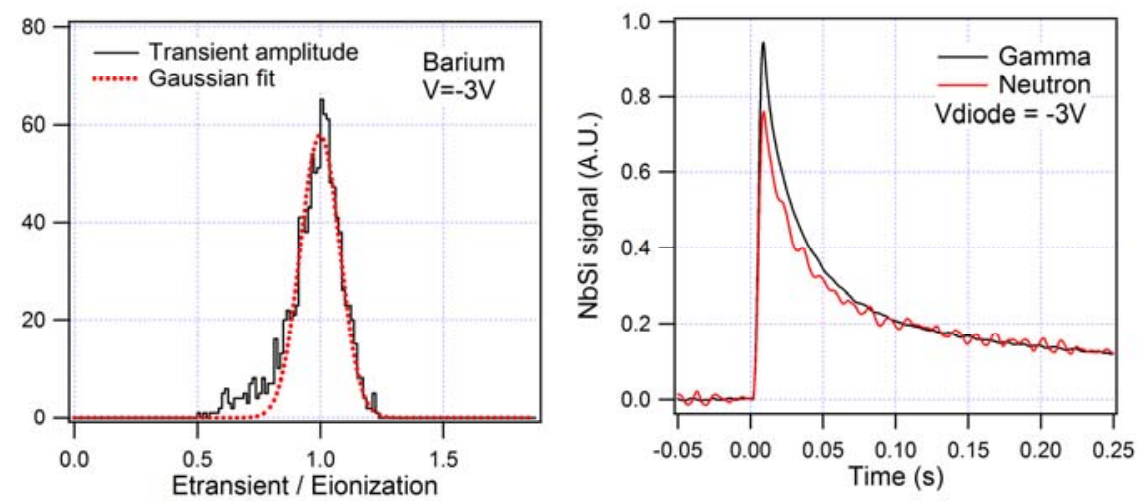

Fig. 3. (left) (Color on line) Histogram of the transient amplitude, normalized by the ionization signal for $50-200 \mathrm{keV} \gamma$ particles. Near-surface events are not included on the graph. (right) signal recordings, showing the slight difference on the transient/thermal ratio between bulk $\gamma$ events and neutrons.

\section{NEAR-SURFACE EVENT REJECTION}

The difference in the athermal signal amplitude of the two opposite $\mathrm{NbSi}$ sensors gives a powerful criterion to identify near-surface events. This is illustrated using a ${ }^{109} \mathrm{Cd}$ source (figure 4). The source is facing the whole surface of the A NbSi sensor, giving interactions all around the top side of the detector. Event localization is based on the $\kappa$ parameter:

$$
\kappa=\frac{T r_{A}-T r_{B}}{T r_{A}+T r_{B}}
$$

$\operatorname{Tr}_{\mathrm{A}}$ and $\operatorname{Tr}_{\mathrm{B}}$ are the normalized transient amplitudes of the two NbSi sensors.

For bulk events the rejection parameter is close to zero while for nearNbSi-surface events $\kappa$ gets closer to +1 or -1 depending on the deposit side ${ }^{7}$. Eliminating events with $|\kappa|>0.3$ rejects in our case approximately $97 \%$ of 
the near-surface events (22 keV, and electrons). It is interesting to remark that the very near-surface $(<100 \mu \mathrm{m}) 22 \mathrm{keV}, 62.5 \mathrm{keV}$ and $84 \mathrm{keV}$ events have approximately the same average $\kappa$ value, meaning that below $100 \mathrm{keV}$ there is only a weak dependence of the localization parameter on deposited energy. The $88 \mathrm{keV} \gamma$ particles penetrates deeper into the absorber $(2 \mathrm{~mm}$ absorption length) giving $\kappa$ values closer to 0 . The enhancement of the transient amplitude of the signal for near-surface events allows effective identification down to the heat energy threshold.

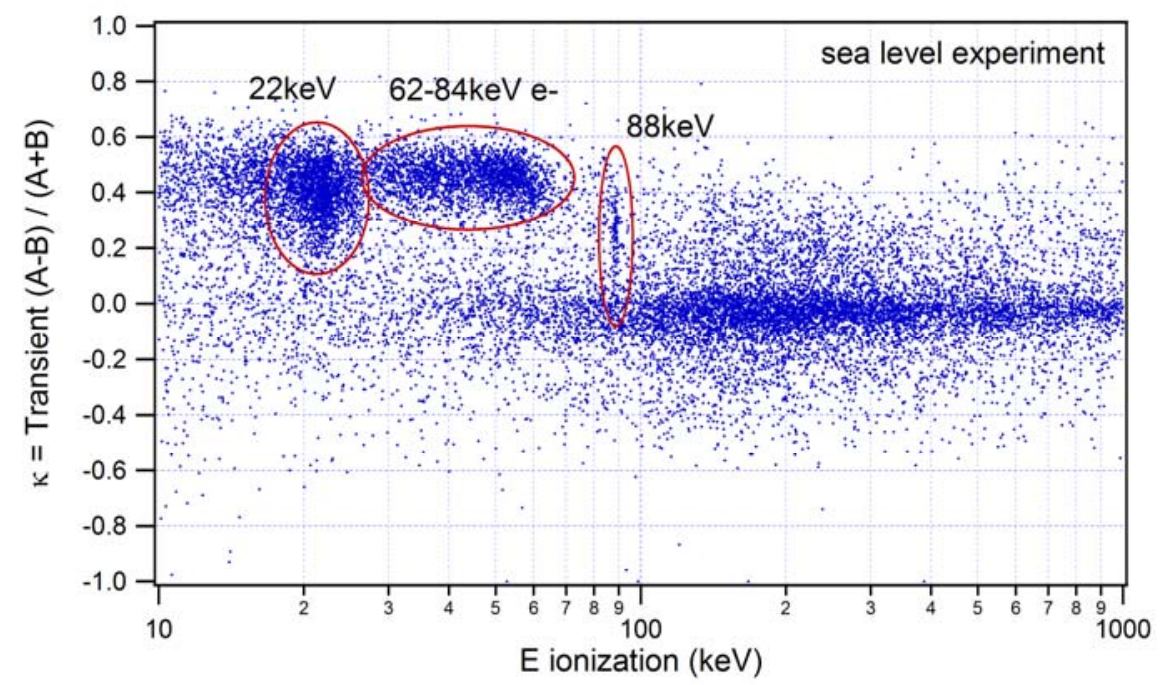

Fig.4. ${ }^{109}$ (Color on line) Cd events are clearly distinguished from the background. The 62.5 and $84 \mathrm{keV}$ electrons show a deficit in ionization energy because of incomplete charge collection.

Based on the neutron calibration run, we can estimate that the $|\kappa|>0.3$ cut eliminates an absorber volume of $2 \mathrm{~mm}$ depth under each $\mathrm{NbSi}$ film. Lowering the limit of the $\kappa$ factor may improve near-surface event rejection but will simultaneously reduce the fiducial volume of the absorber.

During the 2007 low background run in the Laboratoire Souterrain de Modane (LSM) a total of 4.4 kg.d exposure time has been accumulated for the NbSi 212 detector. In addition to the usual plot of the quenching factor based on the thermal part of the heat channel, we can estimate in our case the recoil energy from the transient signal. We consider the mean value of the sensitivity-normalized transient amplitudes, after near NbSi-surface event rejection. The results are shown in figure 5. For the transient based quenching plots we performed a single cut on the localization parameter: 


\section{S.Marnieros et al.}

$|\kappa|>0.3$. The resulting fiducial volume is $34 \%$ of the total crystal size giving $1.5 \mathrm{~kg}$.day effective exposure time. For the thermal signal plots, a further cut on the $E_{\text {transient }} / E_{\text {thermal }}$ ratio was necessary to reject poor quality and pathological heat events resulting in a fiducial volume of $23 \%$ and $1 \mathrm{~kg} . d$ exposure time. Both thermal and athermal signal based quenching plots show no events in the nuclear recoil band after rejection which is very encouraging. The transient based quenching plot seems more interesting because of its higher fiducial volume and its enhanced heat baseline resolution, giving a lower recoil energy limit: $30 \mathrm{keV}$ compared to $70 \mathrm{keV}$ limit. Nevertheless the exposure time is actually too small to conclude to a significant progress with respect to the NTD-Ge detectors of EDELWEISS.
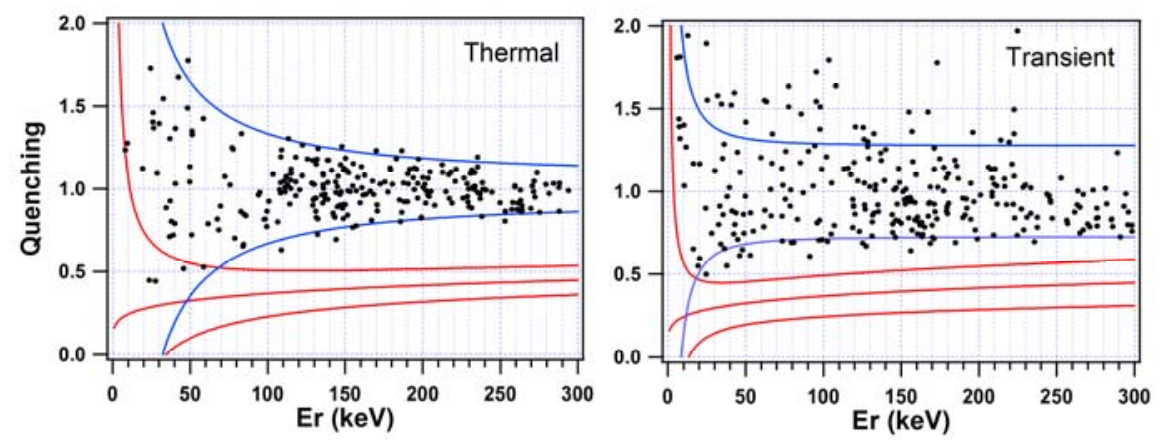

Fig.5. (Color on line) Quenching plots using thermal and athermal heat signal after near-NbSi-surface event rejection. Only pure centre events are selected.

\section{CONCLUSION}

Low background data taking in the LSM using a novel $200 \mathrm{~g} \mathrm{NbSi}$ detector showed no events in the nuclear-recoil band during $1.5 \mathrm{~kg} . \mathrm{d}$ fiducial exposure time above $30 \mathrm{keV}$ recoil energy. Despite the low exposure time of the run, we managed to get very promising results concerning near-surface event rejection capabilities. Transient heat signal modeling allowed a good overall description of the NbSi response.

\section{REFERENCES}

1. T. Shutt et al, Nucl. Instrum. Methods Phys. Res. A 444, 340 (2000).

2. V. Sanglard et al, Physical Review D 71 (2005) 122002

3. D. S. Akerib et al, Phys. Rev. Lett. 96, 011302 (2006)

4. A. Broniatowski et al, LTD12 proceedings (2007) 
5. E. Olivieri et al, LTD12 proceedings (2007)

6. Y. Dolgorouky et al, LTD12 proceedings (2007)

7. S. Marnieros et al, Physica B 259-261 (1999) 862-863

8. B. Neganov and V. Trofimov, Otkrytia i izobreteniya 146, 215 (1985).

9. P. N. Luke, J. Appl. Phys. 64, 6858 (1988). 